\title{
Weak Signals Amplification Through Controlled Bifurcations in Quasi-Two-Dimensional Electron Gas
}

\author{
I. I. Maglevanny， V. A. Smolar, T. I. Karyakina
}

In this paper, we consider the activation processes in a nonlinear bistable system based on a lateral (quasi-two-dimensional) superlattice and study the dynamics of such a system externally driven by a harmonic force. The internal control parameters are the longitudinal applied electric field and the sample temperature. The spontaneous transverse electric field is considered as an order parameter. The forced violations of the order parameter are considered as a response of a system to periodic driving. We investigate the cooperative effects of self-organization and harmonic forcing from the viewpoint of catastrophe theory. Complex nonlinear behaviors including the energetic activation barrier or, more generally, a form of threshold leading to forced bifurcations of dc components of output response accompanied by enhancement of its odd harmonic components were discovered in limited narrow ranges of the control parameters space through numerical simulations. We observed the resonant behaviors of spectral amplification coefficient which is maximized when control parameters are tuned near the critical values of synergetic potential.

Keywords: lateral superlattices, applied electric field, spontaneous transverse electric field, nonequilibrium phase transitions, synergetic potential, forced bifurcations, resonant enhancement

Received August 23, 2018

Accepted November 01, 2018

\author{
Ilya I. Maglevanny \\ sianko@list.ru \\ Tatyana I. Karyakina \\ karyakina@fizmat.vspu.ru \\ Volgograd State Socio-Pedagogical University \\ prosp. Lenina 27, Volgograd, 400066 Russia \\ Vladimir A. Smolar \\ smolar.v@yandex.ru \\ Volgograd State Technical University \\ prosp. Lenina 28, Volgograd, 400131 Russia
}

RUSSIAN JOURNAL OF NONLINEAR DYNAMICS, 2018, 14(4), 453-472 


\section{Introduction}

Activation processes in classical bistable systems in the presence of periodic driving have recently become the subject of growing research activity stimulated by many different scientific disciplines, e.g., biology, electronics and physics.

Analysis of forced vibrations as a response to periodic driving is a major tool for characterizing physical systems and is a very important area of research. Substantial enhancement of input signals based on nonlinear filtering systems has received growing interest due to their promising potential for developing integrated and advanced next-generation nano-devices.

The traditional mechanism for signal amplification is due to nonlinearities in the system transfer characteristics. It should be noted that most of the signal amplification methods studied in the literature are based on effects of stochastic [1] or vibrational resonance [2]. The first effect is when the response of a nonlinear system to a periodic signal is optimized by the presence of a certain amount of noise, the second appears in a nonlinear system driven by two external periodic forces with sharp variation in their frequencies. In this paper we do not consider these effects at the moment and are focusing on a new bifurcation-based scheme that can be employed for robust small-signal amplification (see [3] and references therein). The results bear not only on the fast expanding field of bifurcation-based amplification [4], but also on the physics of classical and quantum fluctuation phenomena in nonlinear vibrational systems, and on the dynamics and fluctuations near bifurcation points.

An important feature of signal amplification through bifurcation is the vibration nonlinearity, which becomes significant even at small vibration amplitudes. It allows investigating a broad variety of nonlinear phenomena, including basic phenomena such as bifurcations, where the number of stable states of the system, or the character of motion, changes with varying parameters. Nonlinear dynamics near bifurcation points is particularly interesting as it is controlled by one, or a few, slow variables [5] and displays universal system-independent features, such as scaling behavior.

Conventional bifurcation amplifiers utilize bifurcations where, as the input changes, the state occupied by the system disappears and the system switches to a different state. In the vicinity of bifurcation points, where the external forcing has its greatest influence, the system goes to the state with either large or small output, depending on the input signal.

The artificially grown quasi-two-dimensional structure - the lateral superlattice (2SL) may serve as an example of materials with required properties. The generic model of SLs, considered in $[6,7]$, is composed of cylinders of GaAs imbedded in a very thin epilayer of GaAlAs. The GaAs cylinders are arranged in a two-dimensional square-lattice array within the epilayer. The different parameters, including layout and material, can be changed while still retaining the superlattice nature of the structure. Such semiconductor structure being highly nonlinear in the nonequilibrium state represents a rather suitable model system for investigation of general rules of self-organization and forced response output in complex nonlinear systems.

In this paper we propose the novel signal amplification strategy and consider the potential model, in which the control parameters affect the potential shape separately or jointly. Thus, the system has potential diversity, which benefits the acquisition of better output. For that we follow an approach developed in $[8,9]$ and consider the nonequilibrium electron gas from a viewpoint of catastrophe theory which provides a powerful tool to discover a criterion for stability and instability in a given system in a situation where the nonequilibrium phase transitions (NPTs) occur. In contrast to the previous paper [9] we concentrate on the kinetic regimes at which the 
higher harmonics of output signal are suppressed, i.e., the input signal is not distorted when transferring the system under consideration.

\section{Sample configuration and static current density}

Before the novel signal amplification strategy is proposed, a criterion to evaluate the output signal and system performance is essential, because all the parameters need to be optimized for the best matching system and optimal output. In contrast to the equilibrium case, when studying the nonequilibrium structures the choice of order and control parameters is not defined unambiguously in advance and depends on conditions of experiment. In this context the interconnections of elements of the structure under investigation should be defined.

The system to be considered consists of linear and nonlinear elements and is driven by an a priori known harmonic signal $U(t)=U_{0} \cos \Omega t$. The parameters $U_{0}$ and $\Omega$ are the parametric driving amplitude and driving frequency; these are easily varied in experimental implementations.

In this paper we consider the following model scheme presented in Fig. 1.



Fig. 1. Scheme of inclusion of the applied electric field $E_{x}$ in the 2SL sample under the harmonic forcing $U(t)=U_{0} \cos \Omega t$.

The effects considered in this paper occur when the applied electric field makes an angle $\alpha=45^{\circ}$ with the principal [01]-axis (Fig. 1). This direction is defined as the $O X$-axis. The sample is supposed to be closed-circuited in series with a finite resistor $R$ in the $O Y$ direction.

When the external electric field $\left(E_{x}\right)$ is applied to 2SL sample, it gives rise to a transverse field $E_{y}[10,11]$ which is treated as an order parameter. This order parameter defines the output signal $U_{A B}(t)=L E_{y}$ - the response of the system, where $L$ is the sample length in the $O Y$ direction. The system control parameters are $R, E_{x}$ and $T$, where $T$ is the sample temperature.

Let us define the equation which describes the dynamics of the order parameter. Assume that the period of the modulation signal significantly exceeds the internal relaxation times of the system. Then the continuity equation of the total current (including displacement current) is determined by the second Kirchhoff law for the contour ABCDA for instant current values and has the form

$$
U_{0} \cos (\Omega t)=L E_{y}+S R\left[\frac{\varepsilon}{4 \pi} \frac{\mathrm{d} E_{y}}{\mathrm{~d} t}+j_{y}\right] .
$$


Here the Gaussian system of units is used, $\varepsilon$ is the permittivity of the sample's material, $j_{y}$ is the static current density in the transverse direction (the nonlinear function of the order parameter and control parameters), and $S$ is the section of the specimen by the plane $O X Z$.

For further calculation the static current density in 2SL under given conditions should be defined.

When one uses a tight-binding approximation, the energy band is confined in two directions $[6,7]$ and in the reference frame defined in Fig. 1 the energy spectrum takes the form [9]

$$
\varepsilon(\boldsymbol{p})=\Delta-\Delta \cos \frac{p_{x} d}{\hbar} \cos \frac{p_{y} d}{\hbar} \quad\left(d=\frac{a}{\sqrt{2}}\right) .
$$

Here $2 \Delta$ is the allowed miniband width, $a$ is the SL constant, $p_{x}$ and $p_{y}$ are the components of the carrier crystal momentum $(\boldsymbol{p})$ with respect to axes which are shown in Fig. 1. The motion of the electron along the third direction is considered to be "frozen".

To calculate the current density $(\boldsymbol{J})$ originated from carriers with the dispersion law (2.2), we confine ourselves to the semiclassical $(2 \Delta \gg \hbar / \tau ; e|\boldsymbol{E}| d)$ and single-band approximation $\left(e|\boldsymbol{E}| d \ll \varepsilon_{g}\right)$, where $\varepsilon_{g}$ is the forbidden miniband width and $\tau$ is the mean free time of electrons.

To present the results in dimensionless form, we introduce the measurement units $p_{0}=\hbar / d$, $T_{0}=\Delta / k$ ( $k$ being the Boltzmann constant), $E_{0}=\hbar / e d \tau, j_{0}=n e \Delta d / \hbar$ ( $n$ being the carrier density) and redesignate

$$
\frac{\boldsymbol{p}}{p_{0}} \rightarrow \boldsymbol{p}, \quad \frac{T}{T_{0}} \rightarrow T, \quad \frac{\boldsymbol{E}}{E_{0}} \rightarrow \boldsymbol{E}, \quad \frac{\boldsymbol{\jmath}}{j_{0}} \rightarrow \boldsymbol{\jmath} .
$$

The necessary charge motion equation may be represented as

$$
\frac{\mathrm{d} \boldsymbol{p}}{\mathrm{d} t}=\boldsymbol{E}, \quad \boldsymbol{p}(0)=0,
$$

where time $t$ is measured in units of $\tau$.

We use the Boltzmann kinetic equation with the $\tau=$ const approximation of the collision integral (one of the arguments in favor of such an approximation are the results of [12] in which it has been established experimentally that at $T>40 \mathrm{~K}$ the mean free time $\tau=$ const and does practically not depend on temperature). Then [13]

$$
\boldsymbol{\jmath}(\boldsymbol{E}, T)=C(T) \int_{0}^{\infty} \boldsymbol{v}(\boldsymbol{p}(t, \boldsymbol{E})) e^{-t} d t, \quad \boldsymbol{v}=\frac{1}{\Delta} \frac{\partial \varepsilon(\boldsymbol{p})}{\partial \boldsymbol{p}},
$$

where $\varepsilon(\boldsymbol{p})=\Delta\left(1-\cos p_{x} \cos p_{y}\right), \boldsymbol{v}$ is is the dimensionless charge velocity, $\boldsymbol{p}(t, \boldsymbol{E})$ is the solution of the Cauchy problem (2.4) and $C(T)=\left\langle\cos p_{x} \cos p_{y}\right\rangle$. Here the angular brackets mean the averaging over the equilibrium carrier distribution. In the following we consider the electron gas nondegenerate, then $[10,11]$

$$
C(T)=\frac{I_{1}(1 / 2 T)}{I_{0}(1 / 2 T)}, \quad \lim _{T \rightarrow 0} C(T)=1, \quad \lim _{T \rightarrow \infty} C(T)=0,
$$

where $I_{n}(z)$ is the modified Bessel function.

Thus, we get the normalized current densities in 2SL [9]

$$
j_{y}\left(E_{x}, T, E_{y}\right)=C(T) \frac{E_{y}\left(1+E_{y}^{2}-E_{x}^{2}\right)}{V\left(E_{x}, E_{y}\right)}, \quad j_{x}\left(E_{x}, T, E_{y}\right)=C(T) \frac{E_{x}\left(1+E_{x}^{2}-E_{y}^{2}\right)}{V\left(E_{x}, E_{y}\right)},
$$


where

$$
V\left(E_{x}, E_{y}\right)=\left(1+E_{x}^{2}+E_{y}^{2}\right)^{2}-4 E_{x}^{2} E_{y}^{2} .
$$

To normalize further Eq. (2.1), we introduce the measurement units of conductivity $\sigma_{0}=j_{0} / E_{0}$, time $t_{0}=\varepsilon /\left(4 \pi \sigma_{0}\right)$ and frequency $\Omega_{0}=1 / t_{0}$ and redesignate

$$
\frac{t}{t_{0}} \Rightarrow t, \quad \frac{\Omega}{\Omega_{0}} \Rightarrow \Omega
$$

As a result, we get the normalized equation which defines the forced dynamics of the order parameter

$$
r A_{0} \cos \Omega t=r E_{y}+\frac{\mathrm{d} E_{y}}{\mathrm{~d} t}+j_{y}
$$

where

$$
A_{0}=\frac{U_{0}}{L E_{0}}, \quad r=\frac{R_{c p}}{R}, \quad R_{c p}=\frac{L E_{0}}{S j_{0}},
$$

here the value of $R_{c p}$ has the sense of sample resistance in weak electric fields.

Define the potential function $\Phi$ - the synergetic potential (the generalized function of entropy production $[10,11]$ ) whose meaning is discussed in [9]

$$
\Phi\left(E_{x}, T, r, E_{y}\right)=\frac{1}{2} r E_{y}^{2}+\int_{0}^{E_{y}} j_{y}\left(E_{x}, T, E_{y}^{\prime}\right) d E_{y}^{\prime}=\frac{1}{2} r E_{y}^{2}+\frac{1}{4} C(T) \ln \frac{V\left(E_{x}, E_{y}\right)}{\left(1+E_{x}^{2}\right)^{2}}
$$

and write Eq. (2.10) in the standard gradient form

$$
\frac{\mathrm{d} E_{y}}{\mathrm{~d} t}=-\frac{\partial \widetilde{\Phi}}{\partial E_{y}}=-\frac{\partial \Phi}{\partial E_{y}}+r A_{0} \cos \Omega t,\left.\quad E_{y}\right|_{t=0}=E_{y 0}
$$

Here $\widetilde{\Phi}=\Phi-E_{y} \cdot A_{0} \cos \Omega t$ is the time-dependent modulated potential [14, 15]. Thus, the harmonic forcing violates the static states which are defined by potential $\widetilde{\Phi}$ at $A_{0}=0$.

\section{Nonequilibrium phase transitions}

Consider first the unforced system $\left(A_{0}=0\right)$. Define the vector of control parameters $\boldsymbol{c}=\left\{E_{x}, T, r\right\}$.

Static macrostates of a system are identified as stable critical points of potential function $\Phi$ and may be received as asymptotic $(t \rightarrow \infty)$ solutions of the problem gradient at given values of control parameters and at $A_{0}=0: E_{y}^{s t}=\lim _{t \rightarrow \infty} E_{y}(t)$.

The set of all macrostates in a space $\left\{\left(E_{y}^{s t}(\boldsymbol{c}), \boldsymbol{c}\right)\right\} \subset R^{1} \otimes R^{3}$ represents the attractor of a dynamical system (2.13). The boundaries of attraction regions define the unstable states (repeller).

By fixed values of control parameters the phase trajectory $E_{y}(t)$ as $t \rightarrow \infty$ tends to some branch of attractor depending on the initial value $E_{y 0}$, i.e., the macrostate $E_{y}^{s t}$ may depend on its initial value in a discontinuous way. Thus, at some values of control parameters the system is characterized by multistability. As the control parameters are varied, the crises of attractor branches take place, which leads to bifurcations and nonequilibrium phase transitions (NPTs) which are interpreted as structural reorganizations.

RUSSIAN JOURNAL OF NONLINEAR DYNAMICS, 2018, 14(4), 453-472 
According to methods of catastrophe theory $[16,17]$, the stationary states are identified with the catastrophe manifold $M$ constructed by the critical manifold of $\Phi$ :

$$
M=\left\{\left(\boldsymbol{c}, E_{y}\right) \in R^{3} \otimes R^{1} \mid \partial \Phi / \partial E_{y}\left(\boldsymbol{c}, E_{y}\right)=0\right\} .
$$

Condition (3.1) (the equation of the system state) defines implicitly the parametric representation of order-parameter critical values, i.e., the (multiple-valued) function

$$
\frac{\partial \Phi}{\partial E_{y}}=0 \Leftrightarrow E_{y}^{c r}=E_{y}^{c r}\left(E_{x}, T, r\right)
$$

The stability of critical values with respect to small fluctuations may be defined by the Morse characteristic of potential $\Phi$ - the critical curvature [16, 17]

$$
W^{c r}(\boldsymbol{c})=\left.\frac{\partial^{2} \Phi}{\partial E_{y}^{2}}\right|_{E_{y}=E_{y}^{c r}} .
$$

At $W^{c r}>0$ the critical point $E_{y}^{c r}$ defines the stable state of the system (attractor), and at $W^{c r}<0$, the unstable one (repeller). Under the criticality condition $W^{c r}=0$ the critical point is degenerate.

The bifurcation set (local separatrix) $\mathcal{B} \subset R^{3}$ in the control parameters space $\left(E_{x}, T, r\right)$ is defined by the condition of degeneration of critical points $\left(W^{c r}=0\right)$ and may be determined by elimination of parameter $E_{y}$ from the conditions

$$
\frac{\partial \Phi}{\partial E_{y}}\left(\boldsymbol{c}, E_{y}\right)=0, \quad \frac{\partial^{2} \Phi}{\partial E_{y}^{2}}\left(\boldsymbol{c}, E_{y}\right)=0
$$

Whenever the control parameters vary very slowly, the system is quasi-static and follows the convention of perfect delay $[16,17]$. This is a rule to determine which of the multiple possibilities the system adopts. The state tends to "occupy" a potential minimum. As long as this minimum is nondegenerate, the system is presumed to follow it. Thus, if some path in control parameters space traverses the local separatrix, the reorganization of a system macrostate occurs - the NPT. The local separatrix is the set of confluence points, at which the potential $\Phi$ is not a Morse function and is structurally unstable. By transversal crossover of the local separatrix due to continuous change of parameters the number of static states changes by 2 , one of them being the macrostate. At some direction of traverse some local minimum and maximum become confluent and a degenerate stationary state occurs, which disappears as the parameters are varied further. Under a reverse parameters change two stationary states appear "from nothing". Thus, the local separatrix separates the parameter plane into open regions in which the number of macrostates is constant and every point $\left(E_{x}, T, r\right)$ parameterizes the structurally stable potential function of qualitatively different type.

\section{Analysis of static macrostates}

Define now the critical manifold and the bifurcation set of the potential (2.12) for $r>0$ and $T>0$. 
The critical manifold is determined by the equation of state

$$
\frac{\partial \Phi}{\partial E_{y}}=0 \quad \Leftrightarrow \quad E_{y}\left[r+C(T) \frac{1-E_{x}^{2}+E_{y}^{2}}{V\left(E_{x}, E_{y}\right)}\right]=0 .
$$

From this equation it follows that the trivial solution $E_{y}=0$ is critical for any values of control parameters. The corresponding Morse characteristic is

$$
W_{1}^{c r}=\left.\frac{\partial^{2} \Phi}{\partial E_{y}^{2}}\right|_{E_{y}=0}=r-C(T) \frac{E_{x}^{2}-1}{\left(E_{x}^{2}+1\right)^{2}}
$$

Equating $W_{1}^{\text {cr }}$ to zero, we get in the space of control parameters a set of branch points defined implicitly by the condition

$$
\mathcal{B}_{1}=\{\boldsymbol{c}\}: \frac{r}{C(T)}=\frac{E_{x}^{2}-1}{\left(E_{x}^{2}+1\right)^{2}} .
$$

The points from set $\mathcal{B}_{1}$ parameterize the potential functions for which the degeneration of static states $E_{y}=0$ takes place. If the path in parameter space traverses the set $\mathcal{B}_{1}$, the branching of the solution to Eq. (4.1) occurs: from the branch $E_{y}=0$ the nonzero solutions separate in a continuous, but nondifferentiable way, i.e., a second-order NPT takes place. Thus, we call the set $\mathcal{B}_{1}$ the set of branching points (each branching is analogous to "Curie temperature" in the classical interpretation of equilibrium phase transitions). The second-order NPTs are characterized by structural instability — the phase transition simply disappears under arbitrary small perturbations or may appear at some other point as a first-order NPT [16].

As $r \rightarrow 0$, the set $\mathcal{B}_{1}$ is a pair of planes $\left|E_{x}\right|=1$, the states $E_{y}=0$ are stable for $\left|E_{x}\right|<1$ and unstable for $\left|E_{x}\right|>1$.

The right-hand side of Eq. (4.3) is negative when $E_{x}^{2}<1$ and reaches its maximum equal to 0.125 when $E_{x}^{2}=3$. Thus, when $E_{x}^{2}<1$ or $r / C(T)>0.125$, Eq. (4.3) has no solutions, and from (4.2) should be $W_{1}^{c r}>0$ for any values of control parameters. In this situation, the set of branch points is empty: $\mathcal{B}_{1}=\varnothing$, only the trivial states of the system $E_{y}=0$ are possible. Since $C(T) \leqslant 1$, only such a regime could be realized for $r>0.125$.

If $r / C(T)<0.125$, Eq. (4.3) has two solutions defining the values of the applied field corresponding to the branch points

$$
E_{1}^{2}=\frac{C(T)}{2 r}\left[1-\sqrt{1-\frac{8 r}{C(T)}}\right]-1, \quad E_{2}^{2}=\frac{C(T)}{2 r}\left[1+\sqrt{1-\frac{8 r}{C(T)}}\right]-1
$$

For $E_{x}^{2} \in\left(E_{1}^{2}, E_{2}^{2}\right)$ we have $W^{c r}<0$, so for such $E_{x}$ the trivial states are unstable, and when $E_{x}^{2} \in\left[0, E_{1}^{2}\right) \cup\left(E_{2}^{2},+\infty\right)$, the macrostate $E_{y}=0$ can be realized.

If $r / C(T)=0.125$, we have $E_{1}^{2}=E_{2}^{2}$, and the set $\mathcal{B}_{1}$ is a pair of planes $\left|E_{x}\right|=\sqrt{3}$.

At $E_{y} \neq 0$ the set of degenerate critical points $\mathcal{B}_{2}$ is defined by exclusion of parameter $E_{y}$ from the conditions

$$
\mathcal{B}_{2}=\{\boldsymbol{c}\}: \frac{1}{E_{y}} \frac{\partial \Phi}{\partial E_{y}}=0, \quad \frac{\partial^{2} \Phi}{\partial E_{y}^{2}}=0 .
$$

Such points parameterize the potential functions for which the degeneration of nonzero critical states takes place. 
According to Eq. (4.1), the nontrivial critical value of the order parameter is determined implicitly by the equation

$$
\frac{E_{x}^{2}-1-E_{y}^{2}}{V\left(E_{x}, E_{y}\right)}=\frac{r}{C(T)}
$$

This equation implies a necessary condition for $E_{x}^{2}>1$ and the estimation $E_{y}^{2}<E_{x}^{2}-1$.

We write Eq. (4.6) in a form solved for the order parameter

$$
\begin{aligned}
E_{y}^{2} & =E_{x}^{2}-1-\frac{C(T)}{2 r}+\mu \cdot \sqrt{\frac{C^{2}(T)}{4 r^{2}}-4 E_{x}^{2}}= \\
& =\frac{C^{2}(T)}{16 r^{2}}\left(1-\frac{8 r}{C(T)}\right)-\left(1-\mu \cdot \sqrt{\frac{C^{2}(T)}{16 r^{2}}-E_{x}^{2}}\right)^{2},
\end{aligned}
$$

where $\mu= \pm 1$.

When $r / C(T) \geqslant 0.125$, the right-hand side of Eq. (4.7) is nonpositive for any $E_{x}$, i.e., nonzero critical values of the order parameter can be realized only at $r / C(T)<0.125$ (this same condition determines the existence of unstable trivial states).

A necessary condition for the existence of a solution of Eq. (4.7) is the condition of nonnegativity of the expression under the root sign, i.e., $\left|E_{x}\right| \leqslant 0.25 C(T) / r$.

The Morse characteristic for the equation of state (4.7) is defined by the condition

$$
W_{2}^{c r}=\mu \cdot \frac{4 r^{2} E_{y}^{2}}{C(T)-\mu \sqrt{C^{2}(T)-16 r^{2} E_{x}^{2}}} \sqrt{1-\frac{16 r^{2} E_{x}^{2}}{C^{2}(T)}} .
$$

Thus, any equation of state (4.7) is stable for $\mu=1$ and unstable for $\mu=-1$.

As $r \rightarrow 0$, we have

$$
\left.\lim _{r \rightarrow 0} E_{y}^{2}\right|_{\mu=1}=E_{x}^{2}-1,\left.\quad \lim _{r \rightarrow 0} W_{2}^{c r}\right|_{\mu=1}=\frac{1}{2} C(T) \frac{E_{x}^{2}-1}{E_{x}^{2}}
$$

The condition $W_{2}^{c r}=0$ defines the set $\mathcal{B}_{2}$, whose points parameterize the potential functions for which there is a degeneration of the nontrivial stationary states

$$
\mathcal{B}_{2}=\{\boldsymbol{c}\}:\left|E_{x}\right|=E_{3}(T, r)=\frac{C(T)}{4 r} .
$$

For control parameters from $\mathcal{B}_{2}$ we have $E_{y}^{2}=E_{x}^{2}-2\left|E_{x}\right|-1$, the necessary condition $E_{y}^{2}>0$ has the form

$$
E_{x}>E_{g}=\sqrt{2}+1 \Leftrightarrow \frac{r}{C(T)}<r_{g}=\frac{\sqrt{2}-1}{4} \approx 0.10355 .
$$

Thus, as $r / C(T) \rightarrow r_{g}$, we have $E_{1} \rightarrow \sqrt{2 \sqrt{2}-1}, E_{2} \rightarrow E_{g}$.

The union $\mathcal{B}=\mathcal{B}_{1} \cup \mathcal{B}_{2}$ is called the bifurcation set or local separatrix [16].

At $r / C(T)<r_{g}$ the set $\mathcal{B}_{2}$ defines the region of tristability [9].

In this paper we consider the case of large conductivity of resistor $R$, i.e., $r_{g}<r / C(T)<0.125$. Then $\mathcal{B}_{2}=\varnothing$ and $E_{x}<E_{g}$. 
Under these conditions we have $E_{x}^{2}-1<E_{g}^{2}-1=2 \sqrt{2}+2, C(T) /(2 r)>2 \sqrt{2}+2$ and for $\mu=-1$ the value $E_{y}^{2}$ from Eq. (4.7) is negative; thus, only $\mu=1$ should be considered, and these nonzero states are stable.

It is easy to verify that it follows from Eqs. (4.7) and (4.4) that

$$
\left.E_{y}^{2}\right|_{\left|E_{x}\right|=E_{1,2}}=\frac{E_{1,2}^{4}-6 E_{1,2}^{2}+1+\left|E_{1,2}^{4}-6 E_{1,2}^{2}+1\right|}{2\left(E_{1,2}^{2}-1\right)} .
$$

At $r_{g}<r / C(T)<0.125$ we have $E_{1,2}^{4}-6 E_{1,2}^{2}+1<0$ and at $\mu=1$ it follows that

$$
\left.E_{y}^{2}\right|_{\left|E_{x}\right|=E_{1,2}}=0 \text {. }
$$

Finally, we get that at $r_{g}<r / C(T)<0.125$ and at $\left|E_{x}\right| \in\left(E_{1}, E_{2}\right)$ we have stable nontrivial states

$$
E_{y}^{2}=E_{x}^{2}-1-\frac{C(T)}{2 r}+\cdot \sqrt{\frac{C^{2}(T)}{4 r^{2}}-4 E_{x}^{2}}>0
$$

and unstable states $E y=0$. At $\left|E_{x}\right| \notin\left(E_{1}, E_{2}\right)$ only the stable state $E_{y}=0$ is possible.

The results of computer simulation of the bifurcation set and the critical manifold for $r \in\left(0, r_{g}\right)$ are presented in Fig. 2 .

The local separatrix decomposes the space of control parameters into the open subsets of points which parameterize structurally stable potential functions of qualitatively different types.

In Fig. 2a the icons show the behavior of synergetic potential $\Phi$ at marked points. Local extremes of $\Phi$ define the critical values of $E_{y}^{c r}$ and local minima correspond to macrostates.

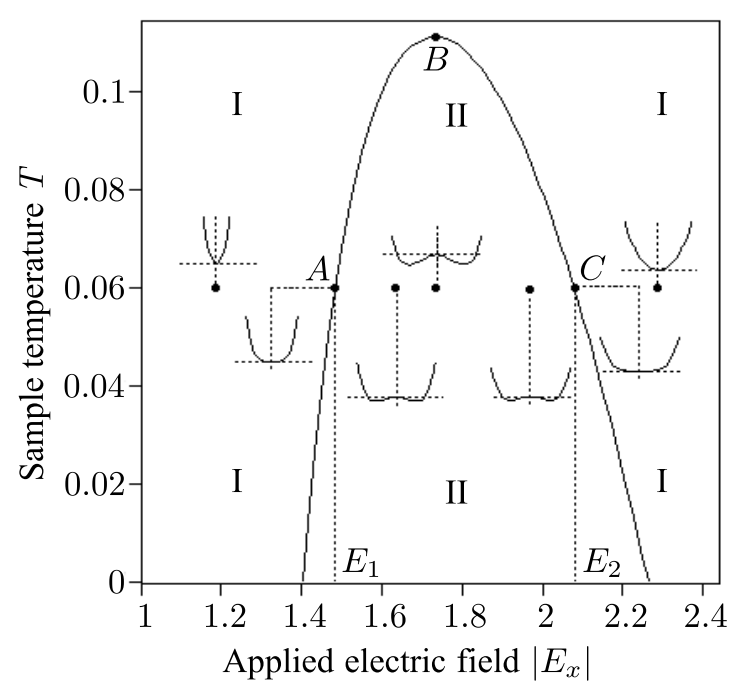

(a)

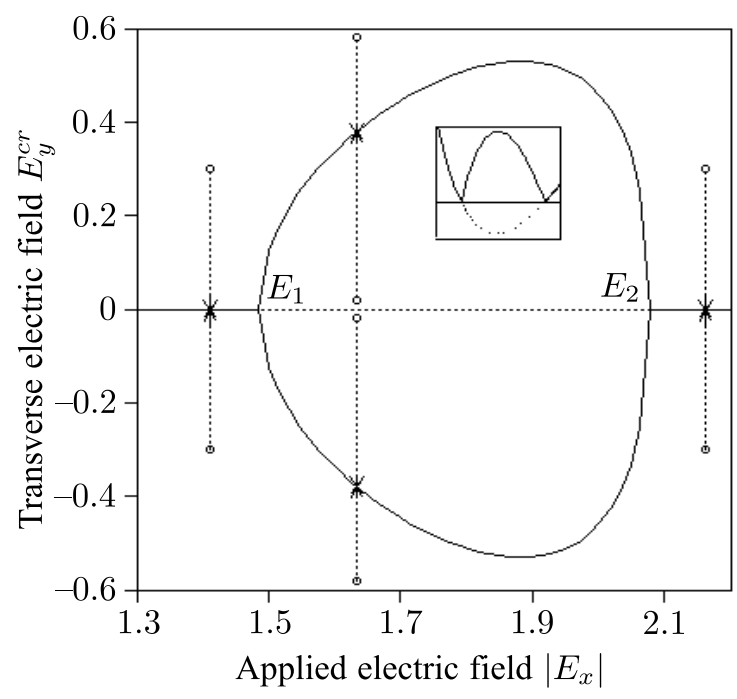

(b)

Fig. 2. Simulation results: (a) the local separatrix $\mathcal{B}_{1}$ on the control parameters plane $\left(E_{x}, T\right)$ for $r=0.11$ (solid lines). At points $A$ and $C$ the central extremes of synergetic potential are degenerate; (b) the critical transverse field (4.7) at $r=0.11, T=0.06$. The solid parts of curves represent the macrostates (attractor), and the dotted parts indicate the unstable states (repeller). Point arrows indicate a phase trajectories, which asymptotically tend to one or another branch of the attractor according to the initial conditions (marked by circles), i.e., the macrostate can depend in a discontinuous way on its initial values. Insert: Morse characteristic $W_{2}^{c r}\left(E_{x}\right)$ (Eq. (4.8)) corresponding to this bifurcation diagram. 
The represented part of the $\left(E_{x}, T\right)$ plane is decomposed into open regions, denoted as I and II, in which potential $\Phi$ has 1 and 2 minima, respectively (the system has an appropriate number of macrostates). In region I the potential has the only trivial macrostate $E_{y}=0$. In region II Eq. (4.7) has two real (nonzero) solutions $E_{y} \neq 0$ located on the upper and lower stable sheets of the critical manifold and corresponding to the local minima of potential $\Phi$. In this case, the state $E_{y}=0$ is unstable.

Along the curves of the local separatrix some local extremes become degenerate. The curve $A B C$ in Fig. 2a is the set $\mathcal{B}_{1}$ bounding the region of bistability. Each point on this curve parameterizes potential functions for which the central minimum is degenerate. Point $B$ corresponding to the maximum of the curve $A B C$ has the coordinates $B\left(E_{\max }, T_{\max }\right)$, where $E_{\max }=\sqrt{3}$ regardless of the value of $r$ and $T_{\max }$ is the solution of the equation $C(T)=8 r$.

For a given region of $E_{x}$ the path $T=0.06$ intersects the local separatrix at two bifurcation points $A, C$, corresponding to abscissas $E_{1}, E_{2}$. At $E_{x} \in\left(E_{1}, E_{2}\right)$ the potential function $\Phi$ has two minimums. The potential well depth and the distance between minimums tend to zero as $E_{x} \rightarrow E_{1}+0$ and $E_{x} \rightarrow E_{2}-0$. Similarly, at fixed $E_{x}=E_{1,2}$ and with varying temperature $T$ the potential function $\Phi$ has two minimums at $T<0.06$, which degenerate as $T \rightarrow 0.06-0$, and when $T>0.06$, the potential function $\Phi$ has only one central minimum. Thus, the potential shape may be controlled both by the applied field and/or by the sample temperature.

Points $E_{1}$ and $E_{2}$ define the turning points of the bifurcation diagram where the switching of the transverse field occurs. One or two macrostates which lie on the stable sheets of the critical manifold may be observed in Fig. 2b, where a bifurcation diagram, characterizing the nonlinear behavior of the system when changing the applied field, is presented.

The multistability of the macrostates (Fig. 2b) at some values of control parameters means that, as they are varied, the crises of the branches of the attractors occur, which lead to bifurcations and NPTs. Thus, for $E_{x}<E_{1}$, there is a single attractor branch (macrostate) $E_{y}=0$. Under a quasi-static increase of $E_{x}$ at $E_{x} \approx E_{1}$ the attractor $E_{y}=0$ turns to a repeller, and a bifurcation of this branch into two symmetric attractor branches $E_{y} \neq 0$ occurs. As a result, a spontaneous symmetry breaking and NPT of the second kind occur, and the locally diagram is similar to the classical bi-fork. It is impossible to predict at which branch (top or bottom) there will be a transition. This is determined by small random perturbations. In the range of $E_{x} \in\left(E_{1}, E_{2}\right)$ there are two symmetric macrostates and one unstable state $E_{y}=0$. The range of $E_{x}>E_{2}$ corresponds to only one stable state $E_{y}=0$. In the reverse change the symmetry breaking occurs at $E_{x} \approx E_{2}$. In the language of semiconductor physics, we are talking about switching effects.

Similar effects appear when you change a parameter $T$ at $\left|E_{x}\right|=$ const [10].

At $r / C(T)>0.125$ the region of bi-stability degenerates, the symmetry macrostate $E_{y}=0$ cannot be violated, and NPTs do not occur. Note also that, as $r \rightarrow 0$, region II is determined by condition $\left|E_{x}\right|>1$, and region I by condition $\left|E_{x}\right|<1$.

\section{Numerical estimation of output response}

For the moment we may view the system as a "black box" and look at how the output power and waveform change as the input signal and system parameters are varied.

The deterministic equation (2.13) describes the evolution of the order parameter under harmonic forcing. This equation was integrated using the embedded fifth-order explicit RungeKutta rule with adapted step-size control based on the Dormand and Prince scheme [18, 19]. 
After the transient processes the system tends to steady state regime, when periodic external influence leads to periodic (nonlinear) fluctuations $E_{y}^{a s}(t)$ of the order parameter around the equilibrium position.

The goal of harmonic studies is to quantify the distortion in power and current waveforms at various kinetic regimes in the system under study.

The primary scope of harmonics modeling and simulation is in the study of periodic, steadystate distortion. Let's represent a system response in the form of Fourier series

$$
E_{y}^{a s}(t)=\bar{E}_{y}^{a s}+\sum_{k=1}^{\infty} A_{k} \cos \left(k \Omega t-\varphi_{k}\right)
$$

Here $A_{k}$ and $\varphi_{k}$ are, respectively, the amplitude and the phase shift of the $k$ th harmonic component defined as

$$
A_{k}=\sqrt{a_{k}^{2}+b_{k}^{2}}, \quad \varphi_{k}=\left\{\begin{array}{l}
\pi / 2, \quad a_{k}=0, b_{k}>0 \\
-\pi / 2, \quad a_{k}=0, b_{k}<0 \\
\arctan \frac{b_{k}}{a_{k}}, \quad a_{k}>0 \\
\pi+\arctan \frac{b_{k}}{a_{k}}, \quad a_{k}<0, \quad b_{k}>0 \\
-\pi+\arctan \frac{b_{k}}{a_{k}}, \quad a_{k}<0, \quad b_{k}<0
\end{array}\right.
$$

the dc value $\bar{E}_{y}^{a s}$ and Fourier coefficients $a_{k}, b_{k}$ are estimated as $[2,20]$

$$
\bar{E}_{y}^{a s}=\frac{2}{m T_{\Omega}} \int_{0}^{m T_{\Omega}} E_{y}^{a s}(t) d t, a_{k}=\frac{2}{m T_{\Omega}} \int_{0}^{m T_{\Omega}} E_{y}^{a s}(t) \cos k \Omega t d t, b_{k}=\frac{2}{m T_{\Omega}} \int_{0}^{m T_{\Omega}} E_{y}^{a s}(t) \sin k \Omega t d t
$$

where $T_{\Omega}=2 \pi / \Omega$ and $m \gg 1$. We include the calculation of values (5.3) in the common scheme. For this we solve the dynamical system

$$
\left\{\begin{array}{l}
\frac{\mathrm{d} E_{y}}{\mathrm{~d} t}=-\frac{\partial \Phi}{\partial y}+r A_{0} \cos (\Omega t),\left.\quad E_{y}\right|_{t=0}=E_{y 0} \\
\frac{\mathrm{d} \bar{E}_{y}^{a s}}{\mathrm{~d} t}=\frac{2}{m T_{\Omega}} E_{y},\left.\quad \bar{E}_{y}^{a s}\right|_{t=0}=0 \\
\frac{\mathrm{d} a_{k}}{\mathrm{~d} t}=\frac{2}{m T_{\Omega}} E_{y} \cos k \Omega t,\left.\quad a_{k}\right|_{t=0}=0 \\
\frac{\mathrm{d} b_{k}}{\mathrm{~d} t}=\frac{2}{m T_{\Omega}} E_{y} \sin k \Omega t,\left.\quad \widetilde{b}_{k}\right|_{t=0}=0
\end{array}\right.
$$

at $t \in\left[0, m T_{\Omega}\right]$.

There are several advantages to using the decomposition in terms of harmonics. Harmonics have a physical interpretation and an intuitive appeal. The number of harmonics to be considered is usually small, which simplifies computation. Consequences such as power amplification can be related to harmonic components and measures of waveform quality can be developed in terms of harmonic amplitudes. 
The most commonly used measure of the quality of a periodic waveform is the total harmonic distortion (THD)

$$
T H D=\frac{1}{A_{1}} \sqrt{\sum_{k=2}^{\infty} A_{k}^{2} .}
$$

When passing the input signal through the nonlinear system the spectrum of the output process is enriched. If the input signal is harmonic, then the periodic modulation of the system leads to periodic perturbation of the potential and of corresponding stationary microstates. As a result, in the spectrum of output signal power, higher harmonics are registered (only odd harmonics in the case of symmetric potential). The amplification coefficient at the $k$ th harmonics due to nonlinearities is characterized by the ratio

$$
\eta_{k}=\left(\frac{A_{k}}{A_{0}}\right)^{2}=Q_{k}^{2}
$$

where $Q_{k}=A_{k} / A_{0}$ is the amplitude response [2].

\section{Forced bifurcations}

For numerical simulations let us define the values of controlled parameters at which the effects of spectral amplification and higher harmonics generation occur. Numerical experiments show that such effects manifest themselves in the vicinity of critical points of modulated synergetic potential and have a distinct threshold character with respect to the amplitude of the input signal as a result of forced bifurcation.

We have confirmed the simulation results within the adiabatic approximation for small driving frequencies $\Omega \ll 1$. All calculations in this section were accomplished at $\Omega=0.001$.

In Fig. 3a the bifurcation diagrams for dc values $\bar{E}_{y}^{a s}$ of output response (see Eq. (5.3)) as a function of the applied electric field are presented. The initial values $E_{y 0}$ for the solution of Eq. (2.13) were chosen in the basin of attraction of the upper branch of the attractor presented in Fig. 2b. At $A_{0} \ll 1$ the dc component coincides with the static macrostate $E_{y}^{s t}$ which is the static attractor. This demonstrates, in particular, the possibility of reconstructing the static critical sets via detection of the dc component of nonlinear response generated by a weak signal.

By variation of the applied field $E_{x}$ the crises of the dc values occur, which lead to bifurcations and NPTs. That is, for $A_{0}=0.01$ and $E_{x}<E_{1}^{t h} \approx 1.64$, there is a single attractor branch $\bar{E}_{y}^{a s}=0$. Under a quasi-static increase of $E_{x}$ at $E_{x} \approx E_{1}^{t h}$ the attractor $\bar{E}_{y}^{a s}=0$ turns to a repeller, and the dc value switches by jump to the attractor branch $\bar{E}_{y}^{a s} \neq 0$. As a result, a spontaneous symmetry breaking and NPT of the first kind occurs. In the reverse change the symmetry breaking occurs at $E_{x} \approx E_{2}^{t h}=1.94$. In the range of $E_{x} \in\left(E_{1}^{t h}, E_{2}^{t h}\right)$ the dc value is near to the static macrostate: $\bar{E}_{y}^{a s} \approx E_{y}^{s t}$.

Thus, at given $E_{x}$ there exists a critical threshold value $A_{0}^{\text {th }}$ at which the dc-component $\bar{E}_{y}^{a s}$ switches from $E_{y}^{a s}$ to zero, and this effect may be interpreted as an attractor escaping by forced bifurcation.

This effect may be simply explained. Consider, for example, a system governed by a double well symmetric static potential $\Phi$. The system is exposed to a harmonic external modulation which is additive. This means that the physical action of the modulation is to "rock" the static potential $\Phi$ to and fro. This has the effect of lowering and raising each potential well with respect to the barrier separating the well. At weak $A_{0}$ the oscillation of the order parameter $E_{y}^{a s}(t)$ 


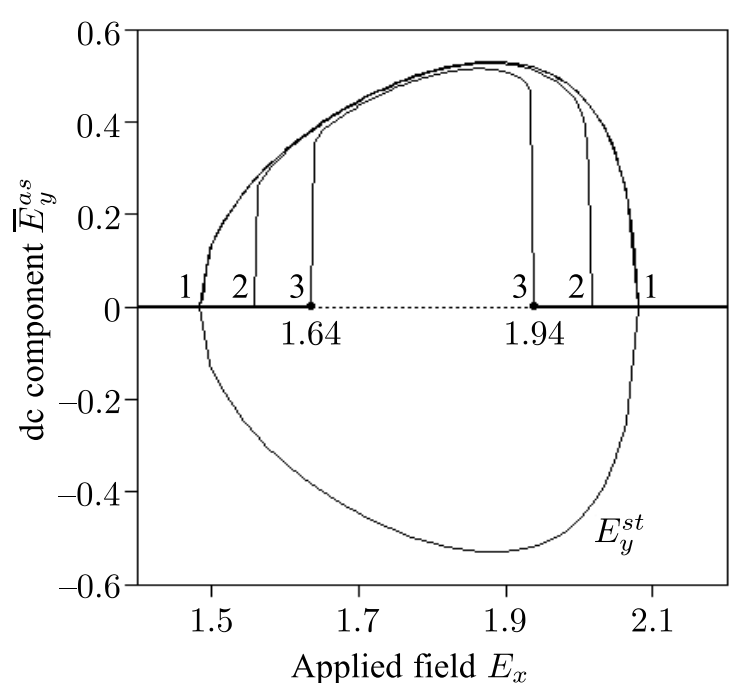

(a)

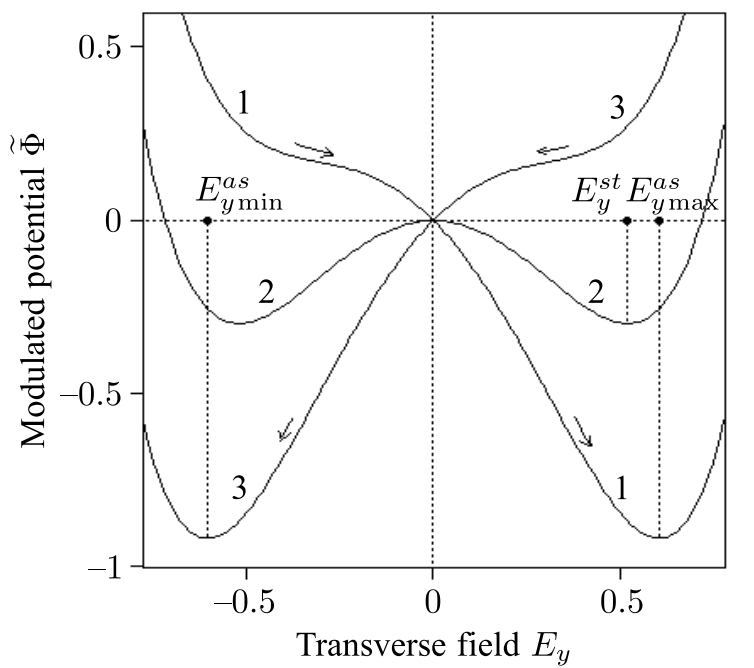

(b)

Fig. 3. Simulation results: (a) bifurcation diagram for the dc value of output response $\bar{E}_{y}^{a s}$ versus $E_{x}$ for some values of $A_{0}$. The curves are: 1) $A_{0}=0.0001$, dc component $\bar{E}_{y}^{a s}$ for this weak input signal coincides with $E_{y}^{c r}$ (see Fig. 2b);2) $\left.A_{0}=0.005 ; 3\right) A_{0}=0.01$. The parameters are: $r=0.11, T=0.06$. Forced bifurcations of the dc-component and switching effects are observed. (b) modulated potential $\widetilde{\Phi}=\Phi+E_{y} \cdot A_{0} \cos \Omega t$ versus the transverse field $E_{y}$. The curves correspond to the following values of $t$ : 1) $t=0$; 2) $t=0.25 T_{\Omega}$, the potential profile coincides with the symmetric static potential $\left.\Phi ; 3\right) t=0.5 T_{\Omega}$; the parameters are: $A_{0}=0.01, r=0.11, E_{x}=1.94, T=0.06$.

occurs around the static macrostate, i.e., $\bar{E}_{y}^{a s}=E_{y}^{s t}$. Thus, the output response is confined to one well. By contrast, when the input voltage is increased, at threshold value $A_{0}^{\text {th }}$, one of the off-center wells disappears and the corresponding minimum of the modulated potential becomes degenerate. According to the convention of perfect delay, the system escapes the well corresponding to the initially chosen attractor $E_{y}>0$ and spends the second half of the period in the other potential well at $E_{y}<0$. This occurs at $t=0$ and $t=0.5 T_{\Omega}$ when $\widetilde{\Phi}=\Phi \mp E_{y} r A_{0}^{\text {th }}$. At such $A_{0}^{\text {th }}$ the forced bifurcation occurs and the dc value of the system output response becomes zero (there will always be a switching event). In contrast, "multiplicative", or "parametric" modulation (by changing the applied field or (and) temperature), as it is sometimes called, has the effect of modulating only the hight of the barrier leaving the relative levels of the two offcenter wells unchanged. Thus, for some combinations of input voltage and internal parameters the above-mentioned forced bifurcation occurs. A schematic representation of this dynamics is shown in Fig. 3b.

The result of forced bifurcation is the periodic switching of output response $E_{y}^{a s}(t)$ between the potential wells; thus, the input signal modulates the system state occupations. This effect is illustrated in Fig. 4. We observe the input signal enhancement which at $E_{x}=1.64$ and $E_{x}=1.94$ (forced bifurcation points) is accompanied by distortion of the output signal wave front, i.e., higher harmonics generation occurs.

The set of points $\left(E_{x}, A_{0}\right)$ on the corresponding parameter plane for which forced bifurcations occur represents the forced bifurcation separatrix, which is presented in Fig. 5a. If some path on the $\left(E_{x}, A_{0}\right)$ plane traverses the forced bifurcations separatrix, forced bifurcations occur and the higher harmonics of output response appear in the vicinity of separatrix points. 


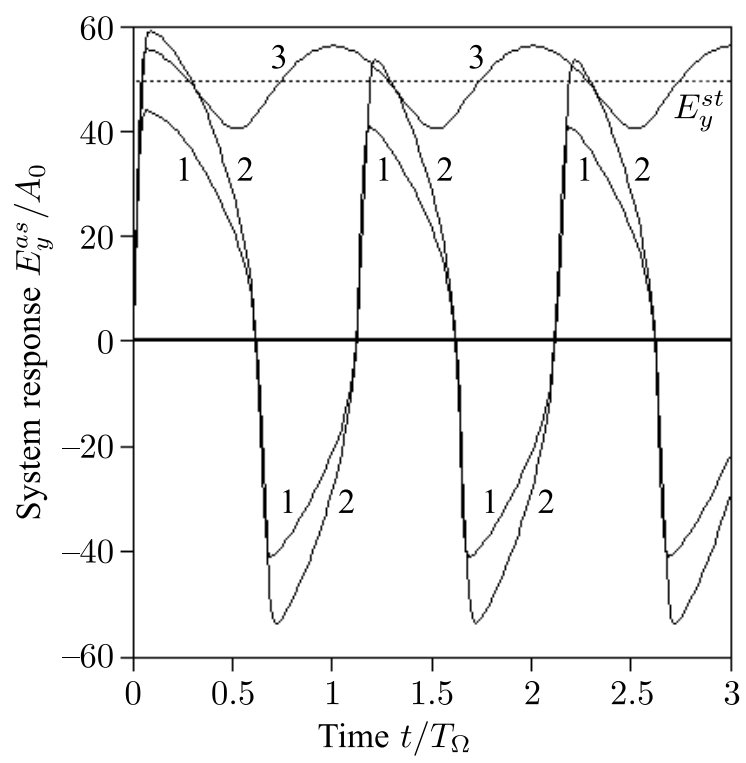

Fig. 4. Simulation results: system response to the input harmonic signal. The curves are: 1) $E_{x}=1.64$; 2) $\left.E_{x}=1.94 ; 3\right) E_{x}=1.79$. The parameters are: $A_{0}=0.01, r=0.11, T=0.06$. Forced bifurcations of dc-component and switching effects are observed.

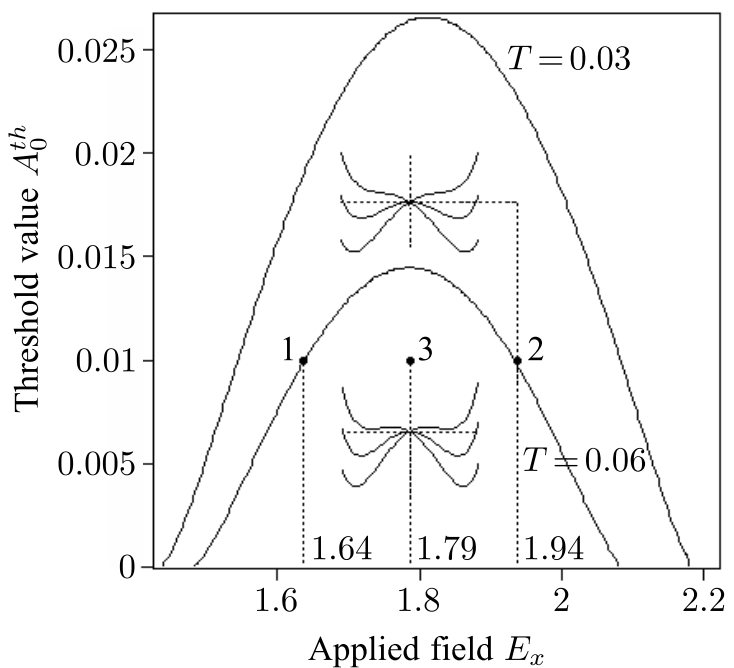

(a)

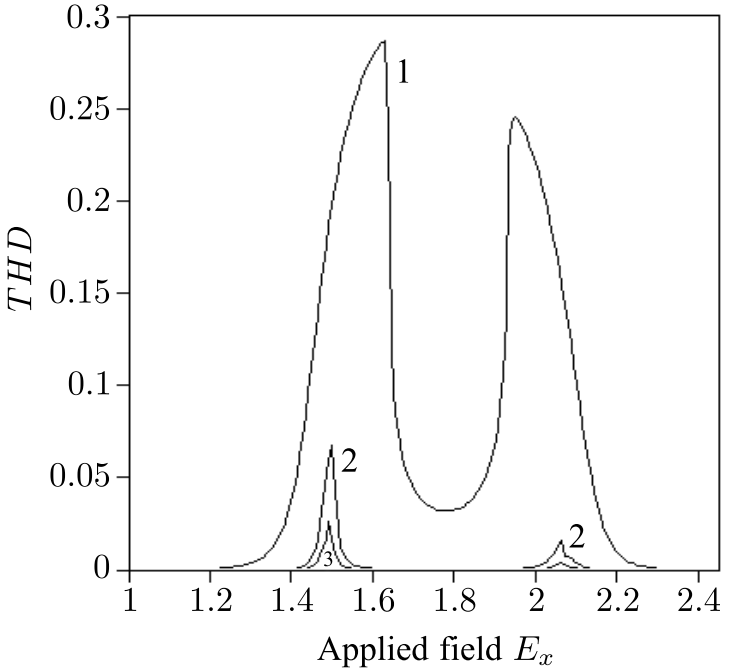

(b)

Fig. 5. Simulation results: (a) the forced bifurcation separatrix on the parameters plane $\left(E_{x}, A_{0}\right)$ at $r=0.11$ for two values of temperature $T$. The inserts show the time-dependent behavior of the modulated potential at marked points. (b) total harmonic distortion versus $E_{x}$. The curves are: 1) $A_{0}=0.01$; 2) $\left.A_{0}=0.005 ; 3\right) A_{0}=0.0005$. The parameters are: $r=0.11, T=0.06$.

For the point 1 (at which $T=0.06, E_{x}=1.94, A_{0}=0.01$ ) the time dependence of the modulated potential is clarified in Fig. 3b. The region between the left and right branches of the separatrix defines the values of parameters at which the effect of higher harmonic generation doesn't show up and the output response is a pure harmonic signal, as illustrated in Fig. 5b.

Therefore, the effect of higher harmonics generation has a distinct threshold character with respect to changing the control parameters and is accompanied by switching of the dc value of output response which may be interpreted as a forced bifurcation. 


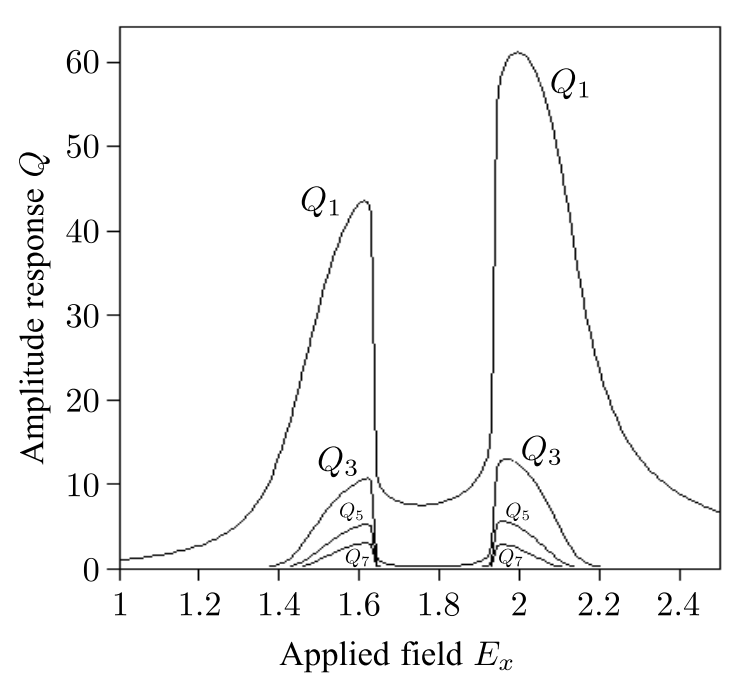

(a)

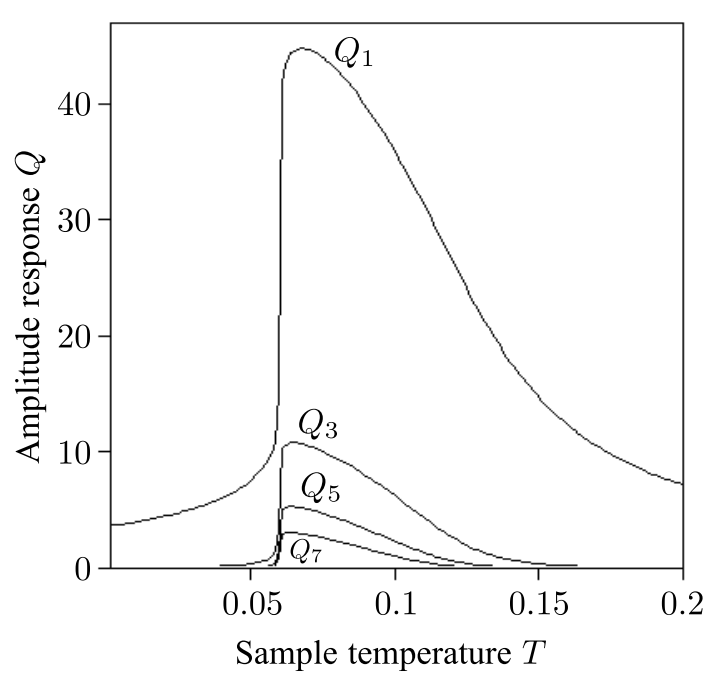

(b)

Fig. 6. Simulation results: (a) amplitude response for four higher odd harmonics $Q_{1}, Q_{3}, Q_{5}$ and $Q_{7}$ versus $E_{x}$. The parameters are: $r=0.11, T=0.06, A_{0}=0.01$. (b) amplitude response for four higher odd harmonics $Q_{1}, Q_{3}, Q_{5}$ and $Q_{7}$ versus $T$. The parameters are: $r=0.11, E_{x}=1.64, A_{0}=0.01$.

In Fig. 6 it is shown that the magnitudes of output response harmonics can be controlled by the applied field $E_{x}$ or by sample temperature $T$. The curves demonstrate resonant behaviors with maximums at points of forced bifurcation.

The curves peaks correspond to points $\left(E_{x}, T, A_{0}\right)$ at the forced bifurcation separatrix presented in Fig. 5a.

As mentioned above, the harmonic forcing violates the static attractor and stimulates the attractor escaping through the forced bifurcations (see Fig. 3a). At very small input voltage $\left(A_{0} \ll 1\right)$ we have $\bar{E}_{y}^{s t} \approx E_{y}^{s t}$. So the detection of the dc component of output response to a weak signal by varying the internal parameters $\left(E_{x}, T\right)$ may provide information about the macrostates of the nonlinear system of interest.

Now we consider the possibility of identifying the static bifurcation set by observation of the system response to weak harmonic forcing. For this, at given $A_{0}$ we calculate the amplification coefficient of output response by varying $\left(E_{x}, T\right)$ and check the values at which the high peaks are observed (these are the points in parameters space at which the forced bifurcations occur). The results of computer experiments are presented in Fig. 7.

The results presented may be interpreted as violations of the static local separatrix by input harmonic forcing. At $A_{0} \ll 1$ these violations are small and the points of forced bifurcations define the points of degeneration of the synergetic potential, i.e., the static bifurcation set.

Thus, we conclude that when weak harmonic forcing is applied to the system under consideration, the observation of output response may provide the identification of critical sets of the synergetic potential of the system.

\section{Weak signal amplification through bifurcation}

For given $T \in\left(0, T_{\max }\right)$, forced bifurcations occur at $E_{x} \in\left(E_{1}, E_{2}\right)$ (see Fig. 2). Consider a situation where $E_{x} \rightarrow E_{1}+0$. Then the threshold value $A^{\text {th }}$ tends to zero, as may be seen in Fig. 8a. 




(a)

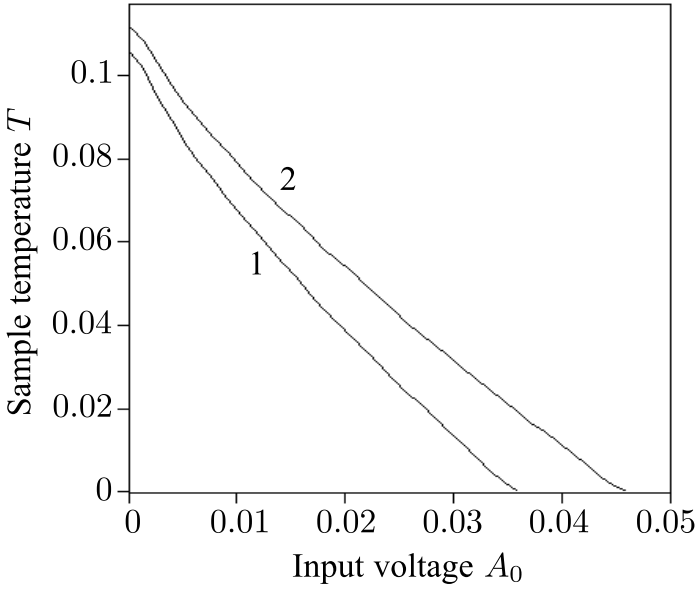

(b)

Fig. 7. Simulation results: (a) points in parameters space at which the forced bifurcations occur for different values of input voltage: 1) $A_{0}=0.0001$, the curve coincides with the local separatrix (see Fig. 2a); 2) $A_{0}=0.005$; 3) $A_{0}=0.01$; $) A_{0}=0.02$; 5) $A_{0}=0.04$. The parameters are: $r=0.11$. With further increase of $A_{0}$ the curves degenerate as shown in Fig. 7b. (b) critical values of temperature $T$ (at which forced bifurcations occur for given $E_{x}$ and $A_{0}$ ) versus $A_{0}$. The curves are: 1) $\left.E_{x}=1.64 ; 2\right) E_{x}=\sqrt{3}$. The parameters are: $r=0.11$.

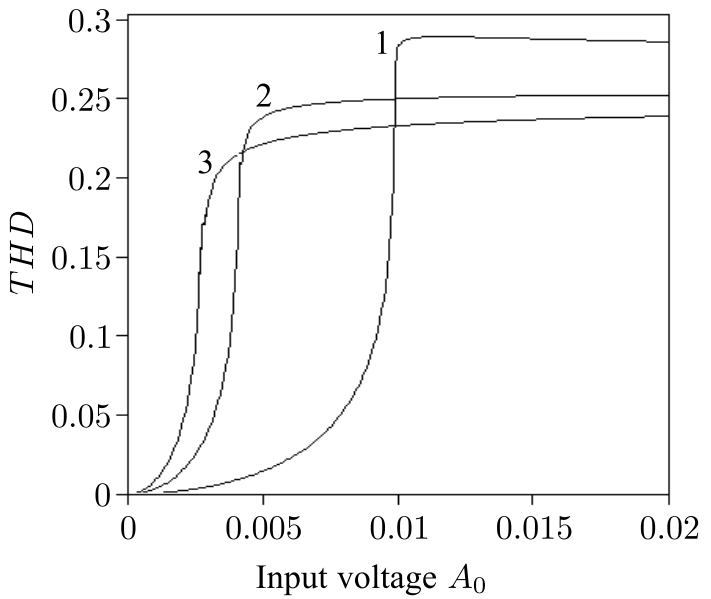

(a)



(b)

Fig. 8. Simulation results: (a) total harmonic distortion versus $A_{0}$. The curves are: 1) $E_{x}=1.64$; 2) $E_{x}=1.55$; 3) $E_{x}=1.53$; the parameters are: $r=0.11, T=0.06, E_{1}=1.4895$. (b) amplitude response for odd harmonics versus $A_{0}$. The parameters are: $r=0.11, T=0.06, E_{1}=1.4895, E_{x}=1.5$.

In this case, the total harmonic distortion $T H D \rightarrow 0$; this means that the output response becomes harmonic, i.e, $Q_{1} \gg Q_{3,5, \ldots}$ and the first harmonic is enhanced. It may be seen in Fig. 8b. Numerical simulations show that the same phenomena occur as $E_{x} \rightarrow E_{2}-0$.

Thus, we may conclude that for a weak input signal $\left(A_{0} \ll 1\right)$ the forced bifurcations occur at values of inner control parameters $E_{x} \approx\left(E_{1,2}, T\right)$, i.e., near the points $A$ and $C$ on the local separatrix (see Fig. 2a). The higher harmonics are suppressed and the approximation of linear response (that is, with linear or "mildly" nonlinear internal dynamics) is valid $E_{y}^{a s}(t) \approx A_{0} Q_{1} \cos \left(\Omega t-\varphi_{1}\right)[1]$ because higher harmonics are practically absent in this case. In addition, $Q_{1} \gg 1$, i.e., signal enhancement is observed. 


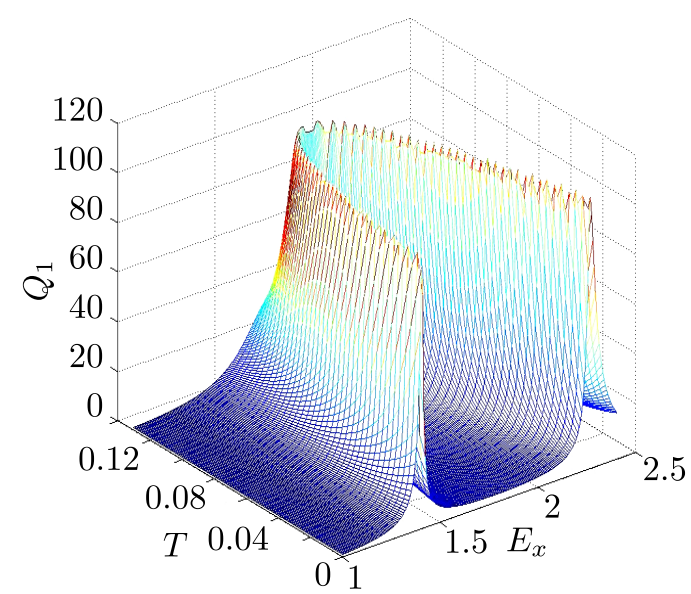

(a)

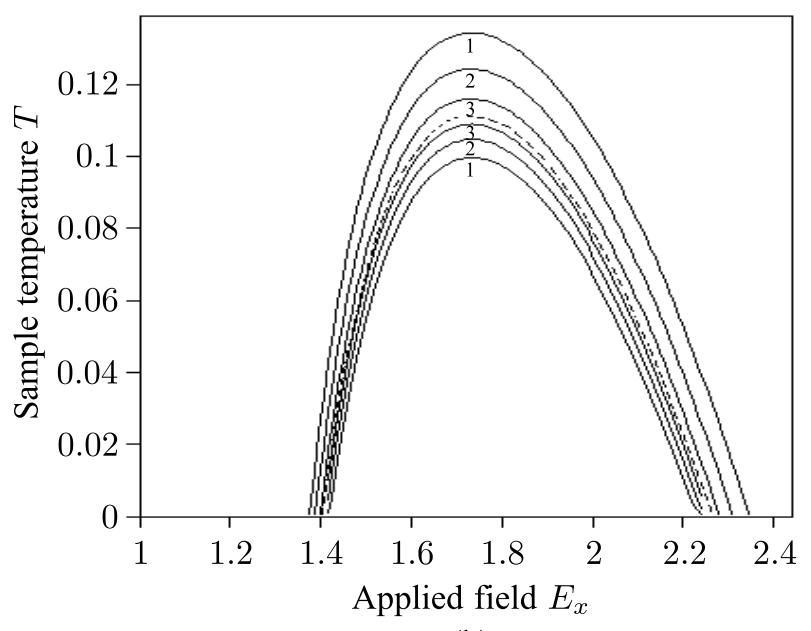

(b)

Fig. 9. Simulation results: (a) the 3D graph of the first harmonic amplitude response $Q_{1}$ is plotted versus the applied field $E_{x}$ and sample temperature $T$. (b) level lines for the function $Q_{1}\left(E_{x}, T\right)$. The curves are: 1) $Q_{1}=30$; 2) $Q_{1}=50$; 3) $Q_{1}=90$. The dashed line is the static bifurcation set. The parameters are: $r=0.11, A_{0}=0.0001$.

Figure 9 displays the 3D surface of the first harmonics amplitude response $Q_{1}$ versus control parameters $\left(E_{x}, T\right)$. One may verify that the maximum of $Q_{1}$ occurs in the vicinity of the separatrix according to Fig. 2a. Note that the maximal values of the spectral amplification coefficient reach considerable values $\eta_{1} \sim 10^{4}$.

As the inner control parameters are varied, the amplification effect manifests a clearly resonant character and is accompanied by a corresponding phase shift of the first harmonic of output response. It is demonstrated in Figs. 10 and 11.

The jumps on these curves occur when the pass in the control parameter space traverses the forced bifurcation separatrix presented in Fig. 5a.

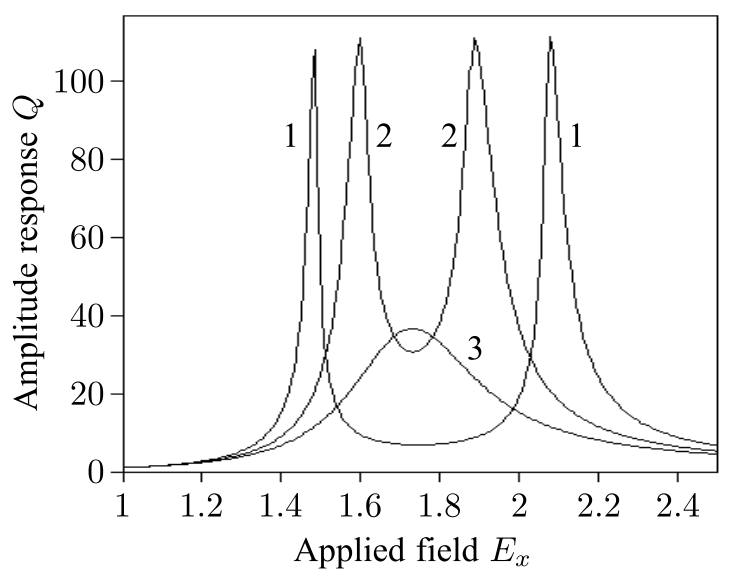

(a)

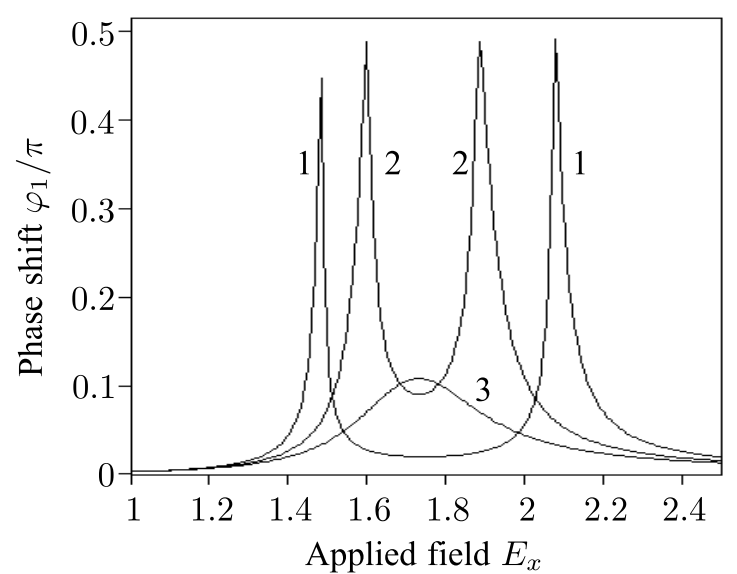

(b)

Fig. 10. Simulation results: (a) amplitude response for the first harmonic $Q_{1}$ versus $E_{x}$ for some values of $T$. The curves are: 1) $T=0.06$; 2) $T=0.1$; 3) $T=0.13>T_{\max }$. The parameters are: $r=0.11$, $A_{0}=0.0001$. (b) phase shift for the first harmonic versus $E_{x}$ for some values of $T$. The curves are: 1) $T=0.06$; 2) $T=0.1$; 3) $T=0.13>T_{\max }$. The parameters are: $r=0.11, A_{0}=0.0001$. 


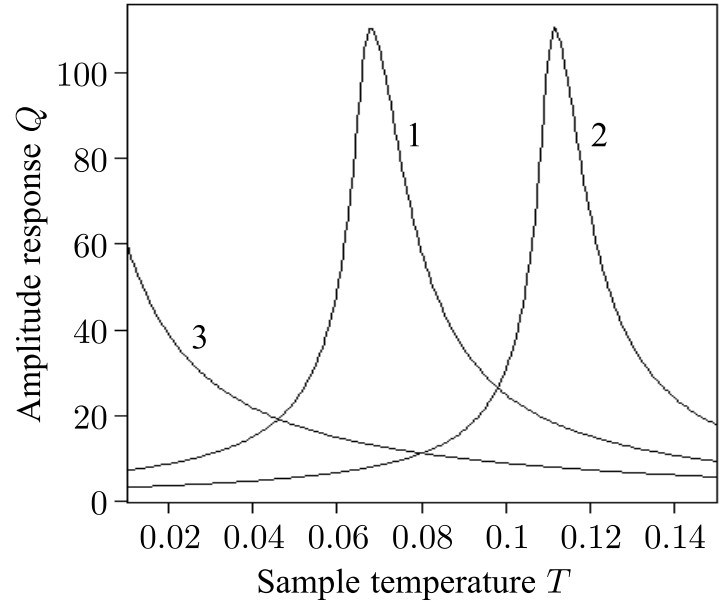

(a)

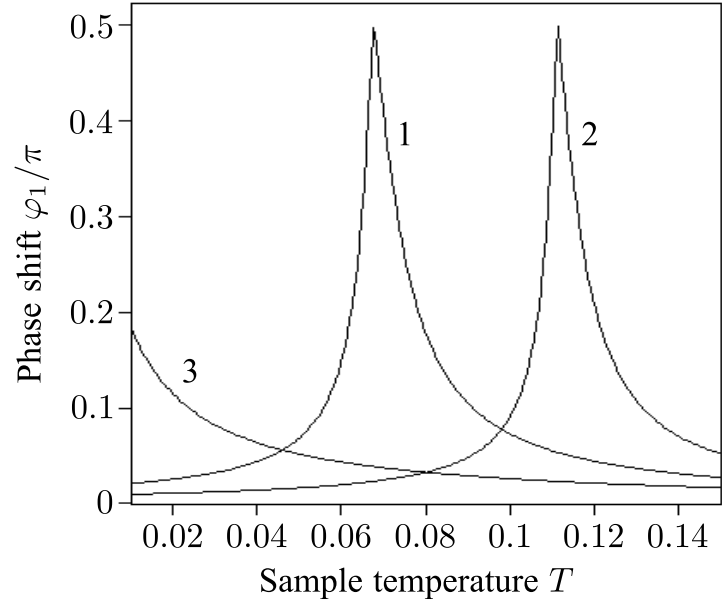

(b)

Fig. 11. Simulation results: (a) amplitude response for the first harmonic $Q_{1}$ versus $T$ for some values of $E_{x}$. The curves are: 1) $E_{x}=1.5$; 2) $E_{x}=\sqrt{3}$; 3) $E_{x}=1.4<E_{1}$. The parameters are: $r=0.11$, $A_{0}=0.0001$. (b) phase shift for the first harmonic versus $T$ for some values of $E_{x}$. The curves are: 1) $\left.\left.E_{x}=1.5 ; 2\right) E_{x}=\sqrt{3} ; 3\right) E_{x}=1.4<E_{1}$. The parameters are: $r=0.11, A_{0}=0.0001$.

\section{Discussion}

In summary, our analysis has discovered the novel phenomena of the parameter-tuning weak signal amplification effect when viewed from the nonequilibrium phase transitions in lateral superlattices. As model simulations show, under the action of harmonic forcing signal one can expect a rich structure of behaviors of output response of quasi-two-dimensional electron gas. A weak signal detection strategy is further proposed and explained in detail by exploring the influences of system output response with different input signals.

The underlying mechanism is fairly simple and robust. The effect requires the following basic ingredients of a system: (i) a coherent input (such as a periodic signal); (ii) a self-organization mechanism that is inherent in the system, and that adds to the coherent input. (iii) an energetic activation barrier or, more generally, a form of threshold. Given these features, the response of the system undergoes resonance-like behavior of the spectral amplification coefficient as a function of control parameters. This is not strictly a resonance in the sense of an increased response when a driving frequency is tuned to the "natural frequency" of the system. In our system we observed the resonant behaviors when the output response is maximized when some parameters - in this case the applied field and sample temperature - are tuned near certain values, namely, the points of symmetry breaking.

Here, some important aspects are summarized and related to computer simulations.

1. By variation of inner control parameters we change the form and number of extremes of the potential function which governs the dynamics of the system, thus controlling the reorganizations of the macrostates of the unforced system (bifurcations).

2. The harmonic forcing violates the static macrostates. The most sensitive are the breaking symmetry points of those values of control parameters for which degeneration of potential function occurs. For a weak input signal $\left(A_{0} \ll 1\right)$ the dc-component of output response coincides with static macrostates: $\bar{E}_{y}^{a s} \approx E_{y}^{s t}$. This means, among other things, that the detection of response to the weak signals may be used for reconstruction of critical values of the synergetic 
potential. Also, the approximation of linear response (that is, with linear or "mildly" nonlinear internal dynamics) is valid $E_{y}^{a s}(t) \approx A_{0} Q_{1} \cos \left(\Omega t-\varphi_{1}\right)$ [1] because higher harmonics are practically absent in this case.

3. A unique synergetic potential model makes it more efficient and effective to adjust the system parameters at which the effect of signal amplification comes up more easily.

4. It is shown that the effect of signal amplification is determined by the forced bifurcation of the dc-component of the output response and this bifurcation has the form of a threshold. In order to get amplification of very high order, we need to adjust the input voltage to the applied field and sample temperature according to the separatrix for forced bifurcations of the dc-component of output response. For the weak input signal $\left(A_{0} \ll 1\right)$ this separatrix coincides with the static bifurcation set of the synergetic potential. This means, among other things, that when weak harmonic forcing is applied to the system under consideration, the observation of output response may provide the identification of critical sets of the synergetic potential of the system.

5. We have demonstrated electric and temperature control of the output response of quasitwo-dimensional electron gas under harmonic forcing and observed the resonant behaviors when the output signal power is maximized when some parameters - in this case the applied field and the sample temperature - are tuned near certain values. The maximal manifestation of the amplification effect occurs along the border of the bistability region, i.e., along the local separatrix.

Numerical estimations of the effects considered are reduced, in general, to measurement units. At $d=10^{-8} \mathrm{~m}, \tau=10^{-12} \mathrm{~s}, \Delta=1.6 \cdot 10^{-21} \mathrm{~J}, n=10^{21} \mathrm{~m}^{-3}$ we get $T_{0} \approx 100 \mathrm{~K}$, $E_{0} \approx 6.6 \cdot 10^{4} \mathrm{~V} / \mathrm{m}, j_{0} \approx 2.4 \cdot 10^{7} \mathrm{~A} / \mathrm{m}^{2}$.

\section{Conclusions}

In recent decades, the great interest in the weak signals detection and amplification is due to a wide range of their possible applications in science and technology.

The parameter-tuning weak signal amplification through bifurcations has great prospects in the applications of signal processing in nonlinear communications systems. In this paper, we report, for the first time to our knowledge, a theoretical and numerical study of the possibility of controlled weak signal amplification through bifurcation by quasi-two-dimensional electron gas.

This paper investigates a new potential model to realize the thermally and electrically controlled amplification effect. This model is based on advanced materials such as lateral superlattices and presents an interesting scenario in which the self-organization effects contribute significantly to the occurrence of signal amplification through bifurcation, a situation that is not found in most familiar models.

The implementation of bifurcation-based amplifier principles, as described here, is based on lateral superlattices. Here we offer the first detailed study of the frequency response of these lattices, as well as a method designed to find the values of system parameters that result in signal amplification. The crux of the method is to locate regions in control parameters space where the output response is intensified. These findings are supported by extensive numerical simulations.

The results of this paper demonstrate that the interplay of external driving and selforganization leads to the onset of specific spectral features in the output power spectra of nonlinear systems. Thus, investigations in the region of self-organization open up possibilities

RUSSIAN JOURNAL OF NONLINEAR DYNAMICS, 2018, 14(4), 453-472 
for the creation of fundamentally new physical devices. We believe that the simple and generic principles underlying the phenomenon considered suggest its applicability to a wide variety of physical systems, such as laser cavities, superconducting resonators, coupled Josephson junctions, and possibly even to oscillating chemical and biological systems.

\section{References}

[1] Gammaitoni, L., Hänggi, P., Jung, P., and Marchesoni, F., Stochastic Resonance, Rev. Mod. Phys., 1998, vol. 70, no. 1, pp. 223-287.

[2] Landa, P. S. and McClintock, P. V.E., Vibrational Resonance, J. Phys. A, 2000, vol.33, no.45, pp. L433-L438.

[3] Karabalin, R. B., Lifshitz, R., Cross, M. C., Matheny, M. H., Masmanidis, S. C., and Roukes, M. L., Signal Amplification by Sensitive Control of Bifurcation Topology, Phys. Rev. Lett., 2011, vol. 106, no. 9, 094102, 4 pp.

[4] Vijay, R., Devoret, M. H., and Siddiqi, I., Invited Review Article: The Josephson Bifurcation Amplifier, Rev. Sci. Instrum., 2009, vol. 80, no. 11, 111101, 17 pp.

[5] Guckenheimer, J. and Holmes, Ph., Nonlinear Oscillations, Dynamical Systems, and Bifurcations of Vector Fields, 5th ed., Appl. Math. Sci., vol. 42, New York: Springer, 1997.

[6] Jafrate, G. J., Ferry, D., and Reich, R., Lateral (Two-Dimensional) Superlattices: Quantum-Well Confinement and Charge Instabilities, Surf. Sci., 1982, vol. 113, nos. 1-3, pp. 485-488.

[7] Reich, R. K., Crondin, R. O., and Ferry, D. K., Transport in Lateral Surface Superlattices, Phys. Rev. B, 1983, vol. 27, no. 6, pp. 3483-3493.

[8] Shmelev, G. M. and Maglevanny, I.I., Transverse EMF in Lateral Superlattices, Physics of LowDimensional Structures, 1996, vol. 9-10, pp. 81-88.

[9] Maglevanny, I. I., Smolar, V.A., and Karyakina, T. I., Thermally and Electrically Controllable Multiple High Harmonics Generation by Harmonically Driven Quasi-Two-Dimensional Electron Gas, Superlattices Microstruct., 2018, vol.118, pp. 29-44.

[10] Epshtein, E. M., Shmelev, G. M., and Maglevanny, I. I., Ferromagnetic and Ferroelectric Properties of Nonequilibrium Electron Gas, Phys. Lett. A, 1999, vol. 254, nos. 1-2, pp. 107-111.

[11] Maglevanny, I. I., Highly Nonlinear Phenomena of Self-Organization of Quasi-Two-Dimensional Electron Gas in High Magnetic and Electric Fields, Phys. Status Solidi B, 2009, vol.246, no.6, pp. 1297-1305.

[12] Grahn, H.T., von Klitzing, K., Ploog, K., and Döhler, G.H., Electrical Transport in NarrowMiniband Semiconductor Superlattices, Phys. Rev. B, 1991, vol. 43, no. 14, pp. 12094-12097.

[13] Bass, F. G. and Tetervov, A. P., High-Frequency Phenomena in Semiconductor Superlattices, Phys. Rep., 1986, vol. 140, no. 5, pp. 237-322.

[14] Jung, P. and Talkner, P., Suppression of Higher Harmonics at Noise Induced Resonances, Phys. Rev. E, 1995, vol. 51, no. 3, pp. 2640-2643.

[15] Contemporary Problems in Statistical Physics, G. H. Weiss (Ed.), Philadelphia: SIAM, 1994.

[16] Gilmore, R., Catastrophe Theory for Scientists and Engineers, New York: Dover, 1993.

[17] Poston, T. and Stewart, I., Catastrophe Theory and Its Application, London: Pitman, 1978.

[18] Press, W.H., Teukolsky, S. A., Vetterling, W.T., and Flannery, B. P., Numerical Recipes: The Art of Scientific Computing, 3rd ed., New York: Cambridge University Press, 2007.

[19] Hairer, E., Nørsett, S.P., and Wanner, G., Solving Ordinary Differential Equations: 1. Nonstiff Problems, 2nd ed., rev., Springer Series in Computational Mathematics, vol.8, Berlin: Springer, 1993.

[20] Bendat, J. S. and Piersol, A. G., Random Data: Analysis and Measurement Procedures, 4th ed., New York: Wiley, 2010. 